\title{
Milligram per Milliliter per Meter Squared
}

National Cancer Institute

\section{Source}

National Cancer Institute. Milligram per Milliliter per Meter Squared. NCI Thesaurus. Code C119393.

A unit of concentration equal to milligrams per milliliter, divided by meters squared. 